Supporting Information

\title{
Embryonic Stem Cells Derived-Small Extracellular Vesicles Modulate \\ Regulatory T Cells to Protect against Ischemic Stroke
}

Yuguo Xia,,${ }^{1 \ddagger}$ Guowen $\mathrm{Hu}, \stackrel{2 \ddagger}{2 \ddagger}$ Y Chen,${ }^{3}$ Ji Yuan,, 3 Juntao Zhang, ${ }^{3}$ Sifan Wang, ${ }^{3}$ Qing $\mathrm{Li}^{3}{ }^{3}$ Yang Wang, ${ }^{3 *}$ Zhifeng Deng ${ }^{1 *}$

${ }^{1}$ Department of Neurosurgery, Shanghai Jiao Tong University Affiliated Sixth People's Hospital, Shanghai 200233, China;

${ }^{2}$ Department of Neurosurgery, The Second Affiliated Hospital of Nanchang University, Nanchang 330006, China;

${ }^{3}$ The Institute of Microsurgery on Extremities, Shanghai Jiao Tong University Affiliated Sixth People's Hospital, Shanghai 200233, China.

\$These authors contributed equally

\section{*Correspondence authors:}

Email: zfdeng@sjtu.edu.cn; wangyang63@sjtu.edu.cn 


\section{Supplementary Figures}

A

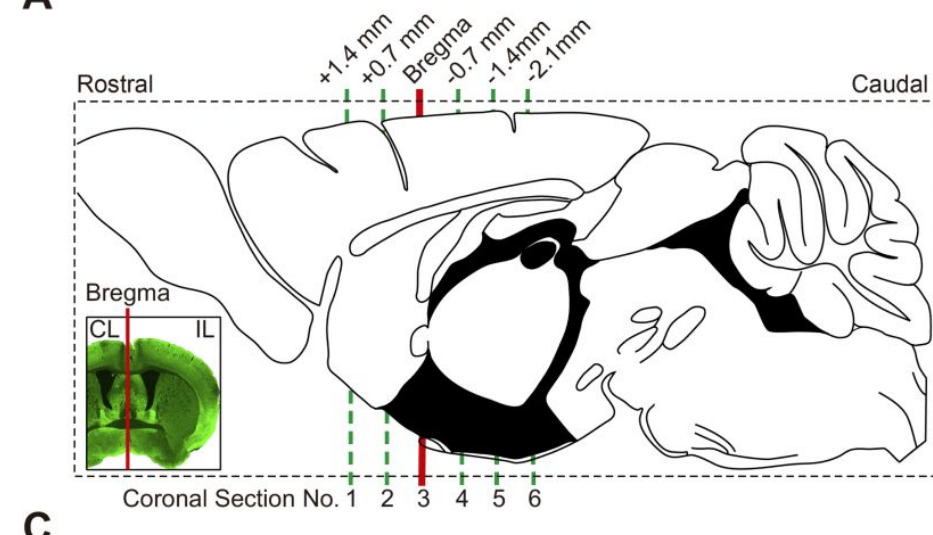

C

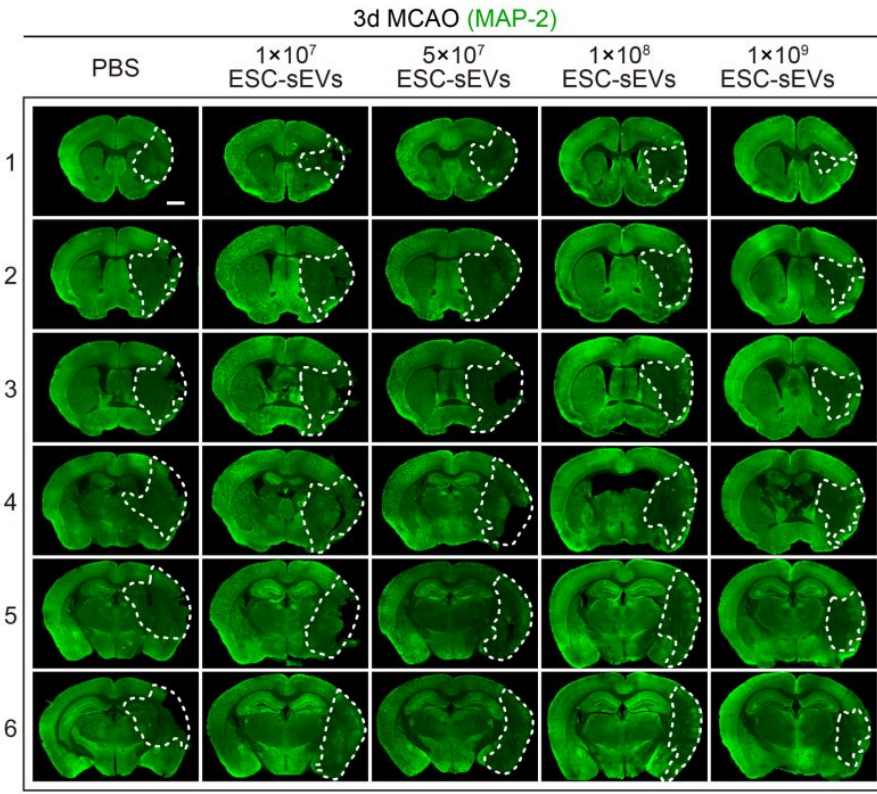

E

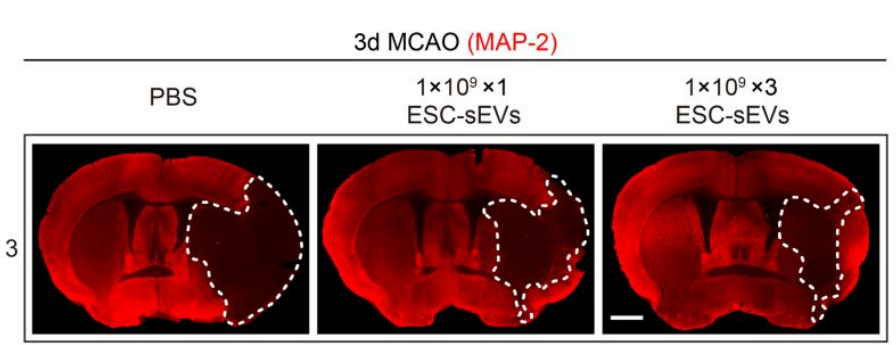

B

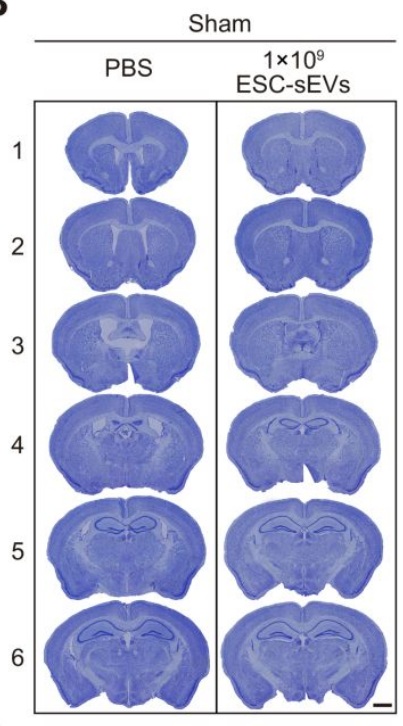

D

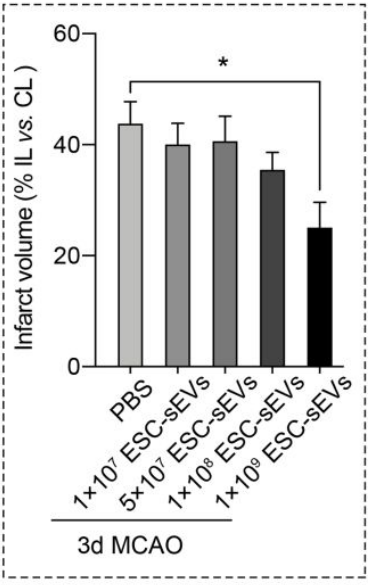

$\mathbf{F}$

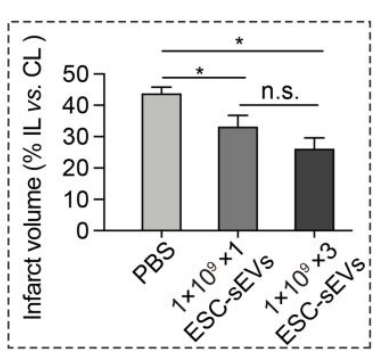

Figure S1. Dose response of ESC-sEVs on stroke therapy. An incremental number of ESC-sEVs particles diluted in $200 \mu \mathrm{L}$ sterile PBS or an equal volume of PBS were injected intravenously for 3 days after MCAO, infarct volume was evaluated by MAP-2 staining of 6 consecutive-coronal slices with an interval of $0.7 \mathrm{~mm}$. (A) Illustration of 
the coronal position of the 6 consecutive slices. CL, contralateral side; IL, ipsilateral side. (B) Representative images of cresyl violet (CV) staining in PBS or ESC-sEVs treated Sham groups. (C) Representative images of MAP-2 staining for the evaluation of infarct volume 3 days after MCAO with different concentrations of ESC-sEVs treatment. Scale bar: 1mm. Dash line, infarct border. (D) Quantification of infarct volume 3 days after MCAO, n=5-7/group. (E-F) PBS, one time, or three times of $1 \times 10^{9}$ ESC-sEVs were intravenously injected starting $2 \mathrm{~h}$ after MCAO procedure. Mice were sacrificed 3 days later for brain infarction measurement. (E) Representative images of MAP-2 staining among groups. Scale bar: 1mm. Dash line, infarct border. (F) Quantification of infarct volume 3 days after MCAO. $\mathrm{n}=8$ /group. ${ }^{*} P<0.05$, n.s. indicates non-significant difference.

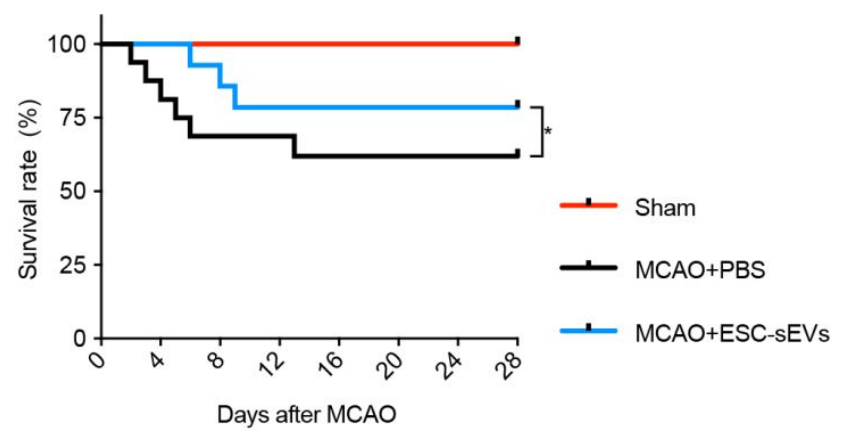

Figure S2. Long-term survival rate after stroke. Mice were intravenously treated with ESC-sEVs or PBS for 3 days, the survival rate was measured up to day 28 after MCAO or Sham operation. ${ }^{*} P<0.05, \mathrm{MCAO}+\mathrm{ESC}-\mathrm{sEV}$ s $v s . \mathrm{MCAO}+\mathrm{PBS}$. 

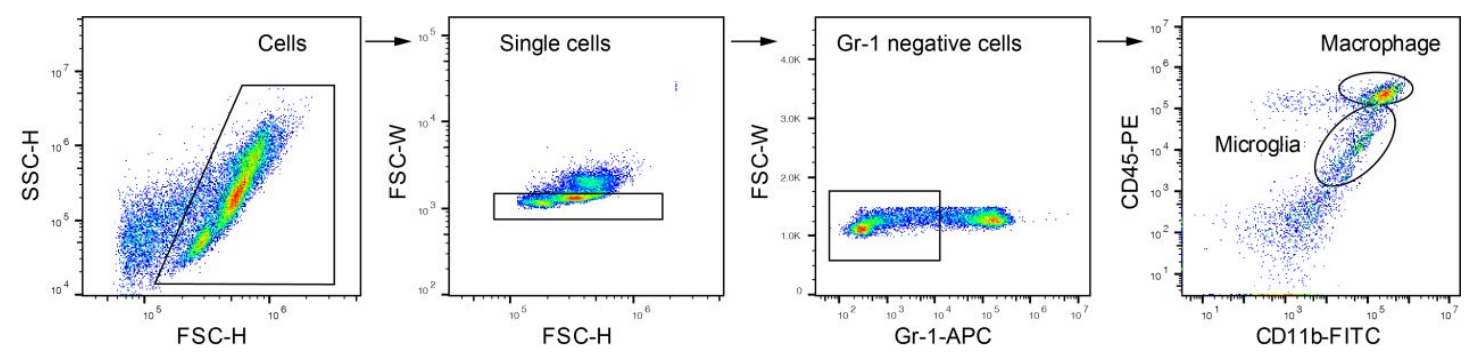

Figure S3. Gating strategy of flow cytometry analysis for $\mathrm{CD}_{11 b^{+}} \mathrm{CD}^{\mathrm{N}} 5^{\text {intermediate }}$ microglia and CD11b ${ }^{+}$CD45 $^{\text {high }}$ macrophage. ESC-sEVs or PBS were administered $2 \mathrm{~h}$ after MCAO, and mice were sacrificed 3 days after MCAO. The ischemic hemisphere was dissected for flow cytometry.

A

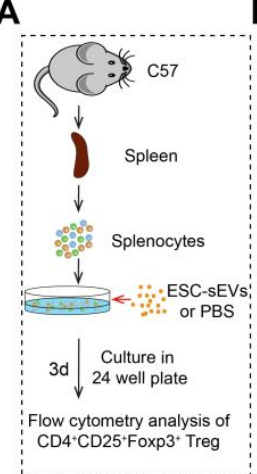

B

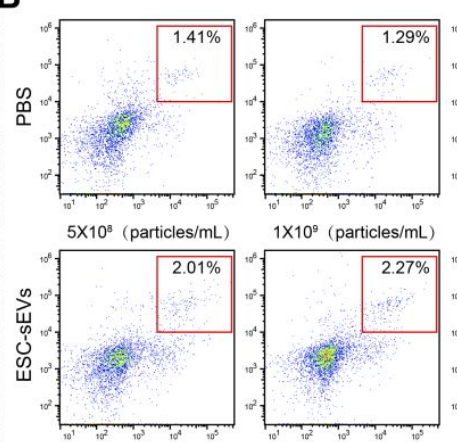

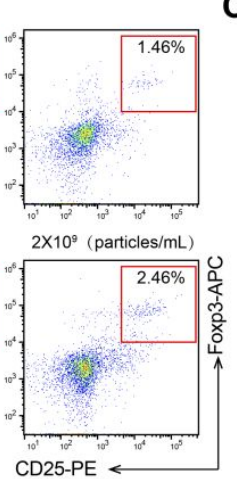

C

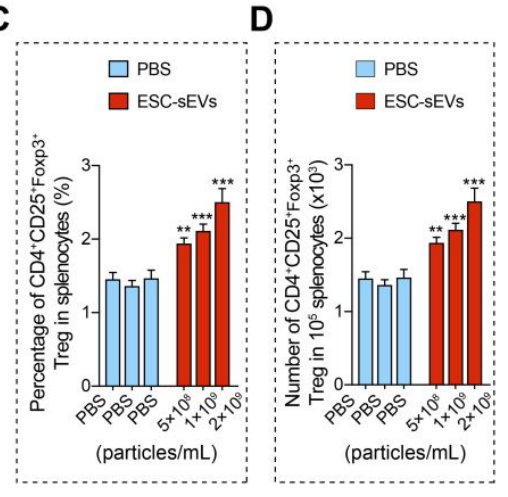

Figure S4. ESC-sEVs treatment expanded Tregs population in splenocytes in vitro.

(A-D) Splenocytes extracted from the spleen of C57/BL6 mice were cultured with the presence of PBS or different concentrations of ESC-sEVs $\left(5 \times 10^{8}, 1 \times 10^{9}\right.$, or $2 \times 10^{9}$ particles $/ \mathrm{mL}$ ) for 3 days. (A) Illustration of the in vitro experimental design. (B) Representative flow cytometry plots for $\mathrm{CD} 4^{+} \mathrm{CD} 25^{+} \mathrm{Foxp} 3^{+}$Tregs. (C) The percentages of $\mathrm{CD}^{+} \mathrm{CD} 25^{+}$Foxp $^{+}$Tregs in splenocytes and (D) the number of 
$\mathrm{CD}^{+} \mathrm{CD} 25^{+}$Foxp $^{+}$Tregs in $10^{5}$ splenocytes, $\mathrm{n}=4$ /group. ${ }^{*} P<0.01,{ }^{*} * * P<0.001$ ESC-sEVs vs. PBS.

A
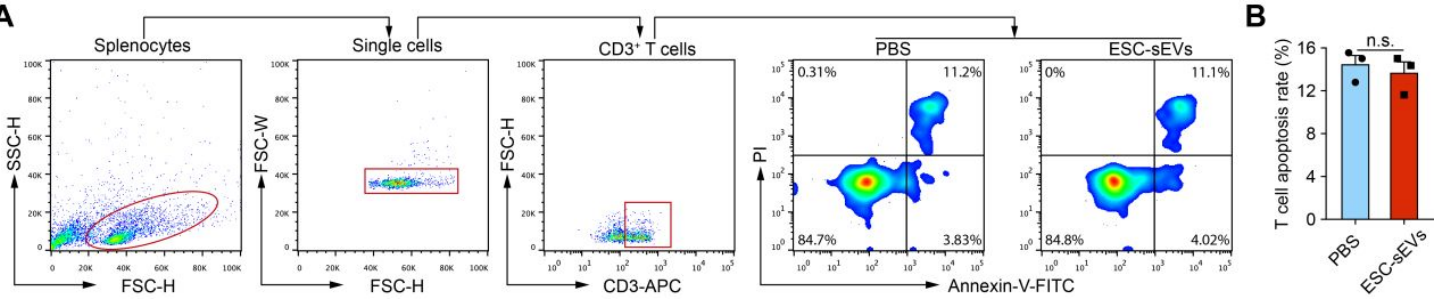

Figure S5. The effect of ESC-sEVs on T cell apoptosis. Splenocytes were cultured in the presence of ESC-sEVs or PBS for 3 days, and the apoptosis rate of $\mathrm{CD}^{+} \mathrm{T}$ cells was assessed by PI and Annexin-V staining. (A) Gating strategy. (B) Quantification of T cell apoptosis rate, $n=3$ /group. $n$.s. indicates non-significant difference.

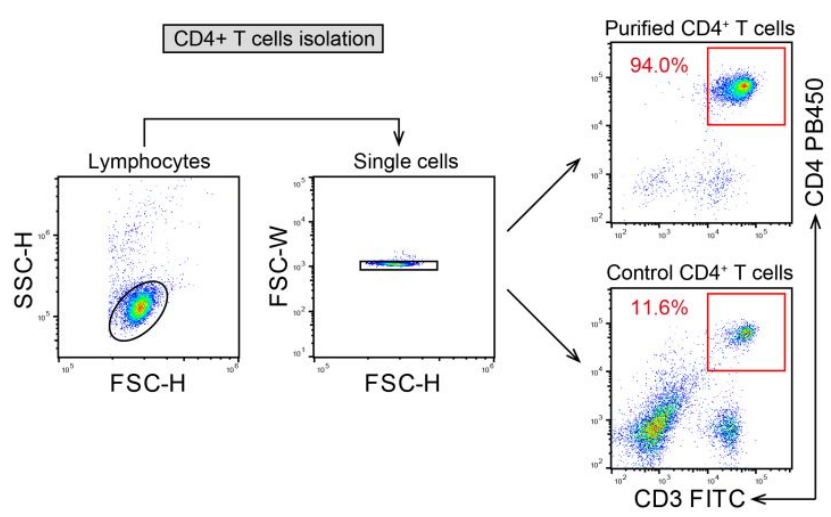

Figure S6. $\mathrm{CD3}^{+} \mathrm{CD4}^{+} \mathrm{T}$ cells isolation. $\mathrm{CD}^{+} \mathrm{T}$ cells were isolated using magnetic according to the operation manual. Flow cytometry analysis revealed that $\mathrm{CD} 3{ }^{+} \mathrm{CD} 4^{+}$ $\mathrm{T}$ cells reached $94 \%$ after purification as compared to $11.6 \%$ in the control group. 

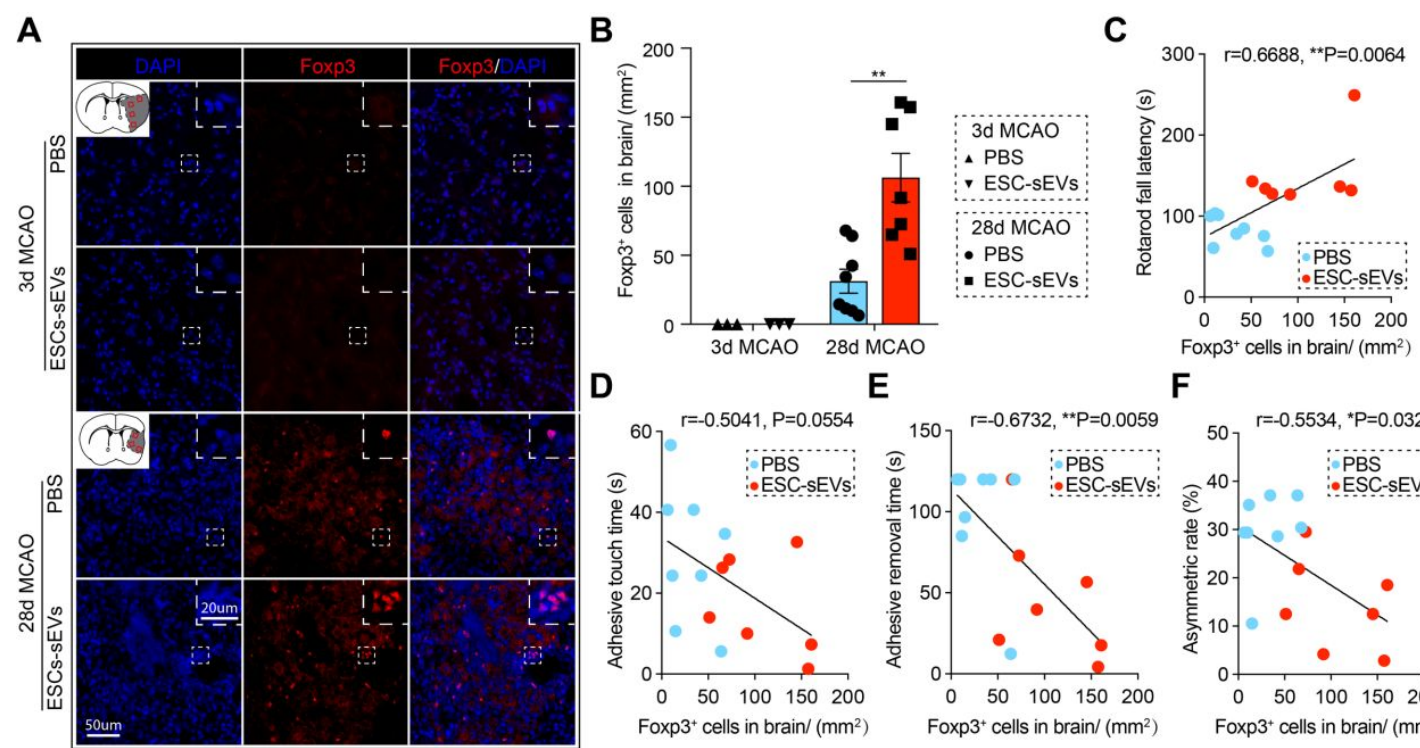

$\mathbf{F}$

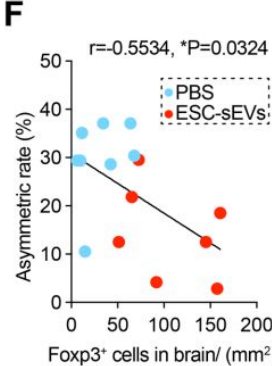

Figure S7. The infiltration of Tregs in brain 3 days and 28 days after stroke. ESC-

sEVs or PBS were administered $2 \mathrm{~h}$ after MCAO, and mice were sacrificed at day 3 or 28 after stroke. (A) Representative immunofluorescence images of Foxp3 (red) staining in the infarct area 3 days and 28 days after MCAO. The red box in the schematics: region of interest (ROI). Scale bar: upper, $20 \mu \mathrm{m}$; lower, $50 \mu \mathrm{m}$. (B) Quantification of Foxp $3^{+}$Tregs in the brain. $\mathrm{n}=3-8$ /group. (C-F) Correlation analysis between the number of Foxp $3^{+}$Tregs and behavior results 28 days after stroke. $\mathrm{n}=7-8$ /group. ${ }^{*} P<0.05,{ }^{*} P$ $<0.01$ ESC-sEVs vs. PBS. 


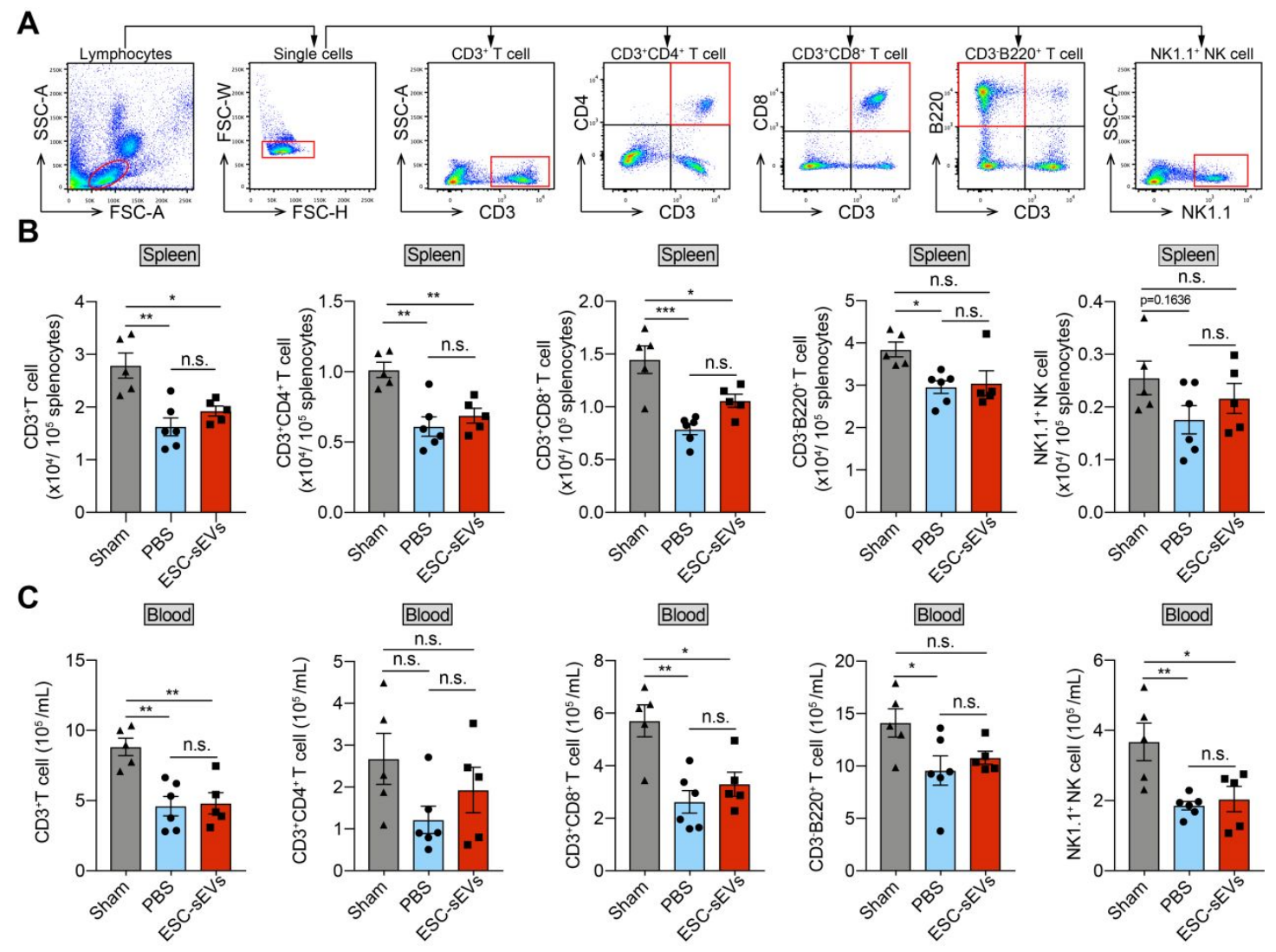

Figure S8. The effect of ESC-sEVs treatment on peripheral immune responses

after stroke. Mice were intravenously treated with ESC-sEVs or PBS for 3 days and flow cytometry analysis was conducted 5 days after stroke. (A) Gating strategy for $\mathrm{CD}^{+} \mathrm{T}$ cells, $\mathrm{CD}^{+}{ }^{+} \mathrm{CD} 4^{+} \mathrm{T}$ helper cells, $\mathrm{CD}^{+}{ }^{+} \mathrm{CD} 8^{+}$cytotoxic $\mathrm{T}$ cells, $\mathrm{CD} 3-\mathrm{B} 220^{+} \mathrm{B}$ cells, and NK $1.1^{+} \mathrm{NK}$ cells in spleen and blood 5 days after MCAO or Sham procedure.

Quantification of immune cells in spleen $(\mathbf{B})$ and blood $(\mathbf{C})$ by flow cytometry analysis, $\mathrm{n}=5-6 /$ group. ${ }^{*} P<0.05,{ }^{*} * P<0.01,{ }^{*} * * P<0.001$. n.s. indicates non-significant difference. 
A

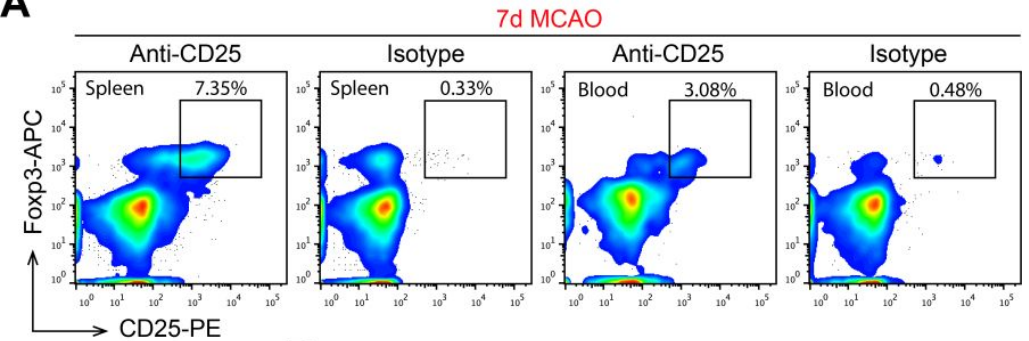

B

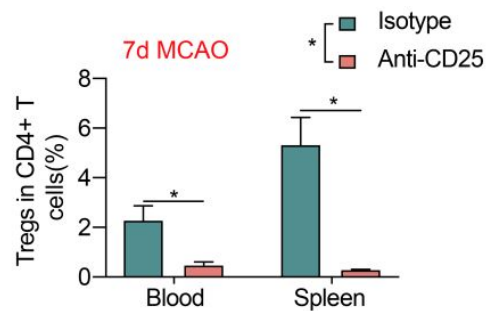

Figure S9. Efficiency of Tregs depletion by anti-CD25 antibody. Anti-CD25 antibody or anti-rat IgG isotype were i.p. injected 48 hours before MCAO, flow cytometry analysis of $\mathrm{CD} 4^{+} \mathrm{CD} 25^{+} \mathrm{Foxp} 3^{+}$Tregs was applied to evaluate depletion efficiency 7 days after MCAO. (A) Representative flow cytometry plots for $\mathrm{CD}^{+} \mathrm{CD} 25^{+} \mathrm{Foxp}^{+}$Tregs in spleen and blood 7 days after stroke. (B) Percentage of Tregs population in $\mathrm{CD}^{+} \mathrm{T}$ cells 7 days after MCAO, $\mathrm{n}=3$ /group. ${ }^{*} P<0.05$, MCAO+Isotype vs. MCAO+Anti-CD25.

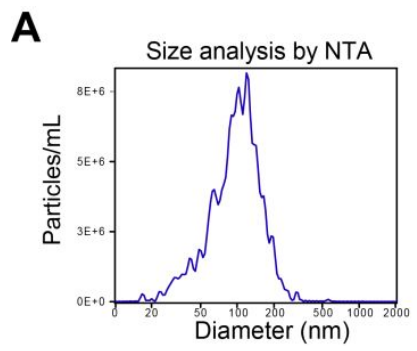

B
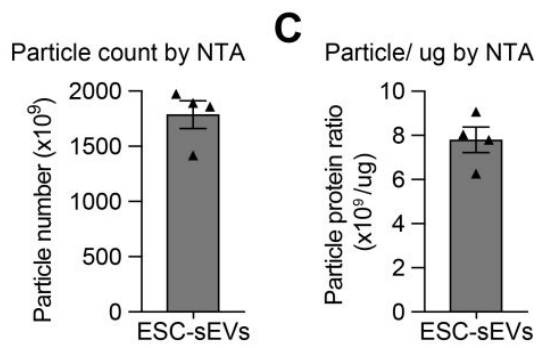

Figure S10. Purity assessment of ESC-sEVs. (A-C) The concentration and size distribution of ESC-sEVs were evaluated by Nanoparticle Tracking Analysis (NTA). (A) Size distribution of ESC-sEVs by NTA. (B) Quantification of the concentration of 
ESC-sEVs by NTA. $n=4$. (C) Particle protein ratio quantification of ESC-sEVs by NTA and BCA analysis. $\mathrm{n}=4$.

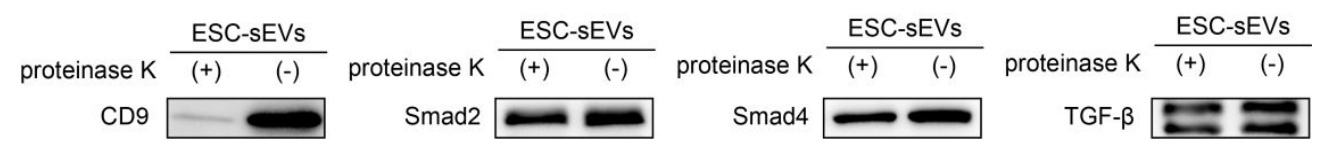

Figure S11. Western blot analysis of proteinase-treated and non-treated ESC-

sEVs. ESC-sEVs were treated with or without $100 \mu \mathrm{g} / \mathrm{mL}$ proteinase $\mathrm{K}$ for $30 \mathrm{~min}$ at $37{ }^{\circ} \mathrm{C}$, and then the proteinase $\mathrm{K}$ was inhibited by incubation with $5 \mathrm{mM}$ phenylmethylsulfonyl fluoride (PMSF) for $10 \mathrm{~min}$ at $37^{\circ} \mathrm{C}$, followed by western blot analysis of CD9 (a surface marker for sEVs), Smad2, Smad4, and TGF- $\beta$.
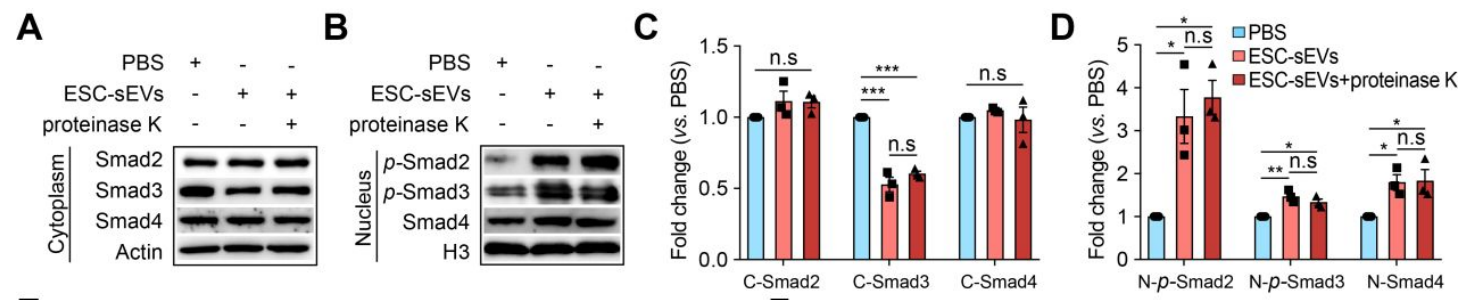

E

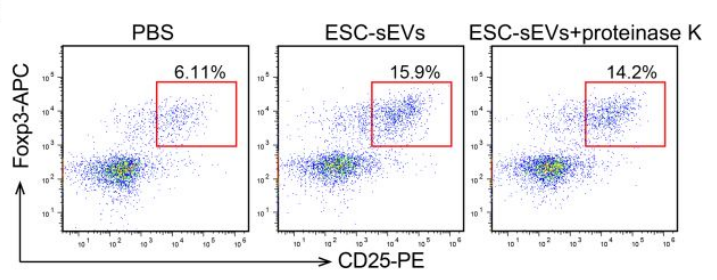

$\mathbf{F}$
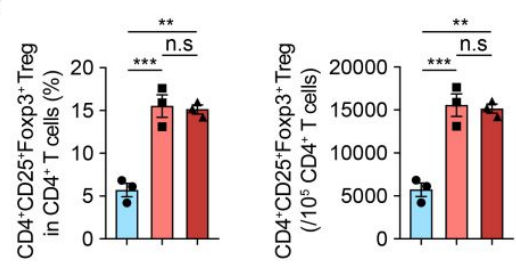

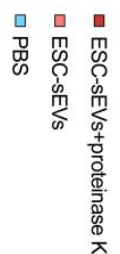

Figure S12. Functional study of proteinase K-treated or non-treated ESC-sEVs.

(A-B) ESC-sEVs were digested with or without proteinase $\mathrm{K}$, followed by ultracentrifugation and resuspension in PBS. Isolated ESC-sEVs $\left(2 \times 10^{9}\right.$ particles $\left./ \mathrm{mL}\right)$ were cocultured with $\mathrm{CD} 4^{+} \mathrm{T}$ cells for 3 days before western analysis of $\beta$-actin, Smad2, 
Smad3, and Smad4 in cytoplasm (A), as well as $p-\operatorname{Smad} 2, p-\operatorname{Smad} 3, \operatorname{Smad} 4$, and histone H3 in the nucleus (B). Quantification of the protein levels in the cytoplasm (C) and nucleus (D). n=3/group. (E) Representative flow cytometry plots for $\mathrm{CD} 4{ }^{+} \mathrm{CD} 25^{+} \mathrm{Foxp}^{+}{ }^{+}$Tregs in $\mathrm{CD} 4^{+} \mathrm{T}$ cells after 3 days of culture with PBS, non-treated ESC-sEVs, or proteinase K-treated ESC-sEVs. (F) Quantification of $\mathrm{CD}^{+} \mathrm{CD} 25^{+} \mathrm{Foxp}^{+}{ }^{+}$Tregs in $\mathrm{CD} 4^{+} \mathrm{T}$ cells. $\mathrm{n}=3$ /group. ${ }^{*} P<0.05,{ }^{* *} P<0.01,{ }^{* * *} P<$ 0.001. n.s. indicates non-significant difference. 